\title{
Toward Effective Hospital Utilization Management
}

\section{Ten Lessons from Case Studies}

\author{
Dean G. Smith, Ph.D.,* and Brenda W. Perry, M.H.S.A.
}

Department of Health Services Management and Policy, School of Public Health, The University of Michigan, Ann Arbor, Michigan

The challenges presented by managed care arrangements and third-party utilization controls have led hospitals to strengthen internal utilization management programs. This article reports findings from a set of case studies on how hospitals have adapted their utilization management programs to their environments. While knowledge acquired from case studies should not be overgeneralized, the findings of these studies suggest some lessons that merit consideration by hospitals advancing their own programs in utilization management.

Utilization management is usually defined as a program to ensure that hospital admissions are clinically appropriate, that medical services are provided in accordance with norms of practice, and that guidelines for reimbursement are followed. The literature contains a growing number of case studies of effective utilization review programs. For example, Mozes et al. (1) found that the application of standardized criteria through daily review at one hospital reduced average length of stay by nearly two days. Catchpole (2) found that the introduction of a utilization management program reduced lengths of stay for $70 \%$ of matched cases. And Woodside et al. (3) found that an intensive review program reduced lengths of stay by almost three days.

Despite these case studies and the great number of articles, books, and guidelines that have been written on how the mechanics of the utilization review process operates, precious little attention has been paid to how the entire utilization management program

\footnotetext{
* Department of Health Services Management and Policy, School of Public Health, The University of Michigan, 1420 Washington Hts., Ann Arbor, MI 48109-2029.

This study was sponsored by the The Mercy Consortium for Health Services Research, Sisters of Mercy, Farmington Hills, Michigan. All statements and opinions expressed are strictly those of the authors and do not necessarily reflect those of the Consortium.
}

should relate to broader hospital strategies, including physician-hospital relations. Do some general principles exist in designing effective utilization management programs, or does each hospital require a unique approach?

As a step toward understanding how various elements of hospital utilization management programs contribute to effective results, case studies of Michigan hospitals were conducted in cooperation with the Sisters of Mercy, Farmington Hills. The purpose of these studies was to identify relevant factors, in addition to the mechanics of utilization review, that contributed to effective operations. Preparing the case studies involved reviewing internal and external reports on utilization experience, interviewing hospital personnel associated with utilization management programs and general administration, and interviewing representatives of third parties involved in hospital reimbursement and utilization review.

Following a brief description of environmental conditions in Michigan common to all study hospitals, findings in five areas of hospital utilization management programs are presented along with ten lessons for effective utilization management. While knowledge acquired from case studies should not be overgeneralized, we believe that these lessons merit consideration by hospitals interested in advancing their own utilization management programs.

\section{THE MICHIGAN HOSPITAL UTILIZATION ENVIRONMENT}

The two largest external utilization management challenges to Michigan hospitals are the Michigan Peer Review Organization (MPRO), working on behalf of Medicare and Medicaid, and Blue Cross and Blue Shield of Michigan's Broad Level Utilization 
Evaluation and Control by Hospital, Inpatient, Physician and Procedure (BLUECHIP) program. Under each of these programs, hospitals are subject to loss of payment for cases judged subacute and may be subject to penalties including much higher compliance costs.

In addition to these two major programs, the Ford Motor Company has its own reporting program for hospitals that admit significant numbers of Ford employees. The Ford reports compare hospital-specific measures of actual use and cost with local area averages (4). Hospitals also interact with literally hundreds of smaller insurers and managed care companies, many of which have their own utilization review protocols. Widely cited reports of the potential of external utilization review programs to reduce costs have encouraged most payers to implement some form of utilization review (5).

In 1988, there were almost 370,000 Medicare hospitalizations in Michigan, averaging 8.9 days and $\$ 5,150$ apiece. With these results, Michigan's average Medicare cost per enrollee ranked among the highest in the country (6). In 1989-1990, approximately $2.1 \%$ of all cases reviewed by MPRO were ultimately deemed to be inappropriate (7). Therefore, third-party involvement and pressure on hospital utilization management may be greater in Michigan than in other states.

Each of the study hospitals was located in an urban area and had over 300 acute care inpatient beds, some teaching programs, and a broad range of acute care services. Since the beginning of the MPRO and the BLUECHIP programs, most study hospitals have achieved average or better results in utilization audits, meeting or exceeding target denial thresholds every period with only one or two exceptions. Ford reports have indicated that study hospitals are not unusually different from area averages for most services. The hospitals are located in different cities and therefore face slightly different mixes of payer groups and utilization requirements.

\section{FIVE CHARACTERISTICS OF UTILIZATION MANAGEMENT PROGRAMS}

To provide a more well developed context for the lessons on effective utilization management, brief findings from the case studies are presented for each of the five general characteristics of utilization management programs.

\section{General Utilization Management Strategy}

A hospital's general strategy is the set of fundamental policies that guide the structure and operation of utilization management activities (8). These policies are typically developed or revised by the hospital's utilization review committee, which includes representatives from quality assurance, medical staff, and administration. Among the most visible elements of a utilization management strategy are the starting point of activity for utilization management and the involvement of physicians.

As has been found in other studies, hospitals revealed a range of strategies in response to pressures from third-party payers (9). The starting point for some hospitals involved active preadmission screening. Teams of nurses armed with admission criteria reviewed all admissions for all payers. For others, utilization review was initiated after admission and consisted of minimal compliance with documentation requirements of selected payers.

Irrespective of the hospitals desired starting point for utilization management, many insurers require precertification for several diagnostic and surgical procedures. Therefore, most hospitals require assistance from physician offices in obtaining precertification information, and many physicians' offices attempt to leave the entire responsibility to the hospital utilization management staff. Some specific precertifications required for admitting patients are usually the sole responsibility of the hospital (e.g., most cardiac catheterizations).

Involvement of physicians in utilization management can take many forms, but it usually starts with the design of the utilization management program itself and determination and education on third-party requirements. Hospital strategies for physician involvement ranged from active education and solicitation of input to passive record-keeping of activities and minimal contact with physician offices. While all physicians interviewed indicated personal familiarity with third-party requirements, utilization management reviewers suggested that such familiarity was the exception and not the norm among medical staff. Given the variation in criteria and insurers' requirements, it may not be necessary that physicians be well versed on requirements, but rather that they be attentive to utilization management reviews on questionable cases and adopt a cooperative spirit toward dealing with the information demands on precertification.

\section{Evaluation Tools and Methods}

The process of utilization management in its most simple form involves matching patient conditions with 
established criteria. Having established criteria that are appropriate and that match those of third-party payers is essential to this process. The large and increasing number of private third-party reviewers, sometimes each with their own criteria sets, and the complexities of identifying the primary insurer have made the task of matching criteria sets to patients more challenging. Although from the hospital's viewpoint there is a great need for a uniform set of criteria, one has not yet emerged, and is not likely to emerge until agreements are reached on reasonable parameters of practice (10). Thus, variations in criteria will persist for at least the next few years, and the ability to comply with a wide range of alternative regulations will remain important.

At study hospitals, the use of evaluation tools generally included some combination of MPRO and BLUECHIP criteria. Evaluations of reliability and validity have found that most common utilization review instruments are acceptable (11). At one hospital, a booklet of combined criteria was assembled and used to evaluate all cases. More typically, only Medicaid, Medicare, and managed care cases that require authorizations were reviewed.

\section{Utilization Management Staff}

The role of the physician as the director of patient care cannot be denied. However, in the literature, the role of physicians in utilization management functions is somewhat unclear. Linton and Peachey (12) suggested that utilization management should be entirely in physicians' purview, while Sutherland (13) argued that physicians are but one interested party and perhaps not in the best position to perform all utilization management functions. Connors (14) reported that physicians are becoming more willing to participate in patient planning, including working with utilization management personnel.

Utilization management programs at study hospitals were staffed by combinations of nurses, medical records personnel, and other hospital employees; each type of background offers a unique set of strengths and weaknesses. Nurses were viewed as having more credibility with physicians for questionable cases and as having a better understanding of the medical significance of criteria. Medical records personnel were thought to have a better understanding of the medical record, its coding, and interpretation. Other persons without these medical backgrounds were considered to be more objective at applying criteria and less expensive to employ. The relative efficiencies of nurses, medical records, and other personnel have not been subject to evaluation, but they did not appear to vary among the study hospitals. On average, reviewers dedicated to utilization management processed an estimated 25-30 cases per day, in addition to placing calls to third parties. Reviewers with substantive assignments in quality assurance, infection control, and risk management performed slightly fewer reviews.

\section{Physician Advisor Role}

"A good physician advisor is a precious asset" (15). Historically, physician advisors have been volunteer, rotating members of a utilization management or quality assurance committee. More recently, hospitals have started to pay advisors and several have hired nonmedical staff consultants to act as advisors.

In a typical hospital program, upon an initial comparison of a chart with criteria, the nonphysician reviewer either approves the admission or calls into question the necessity of admission. Questionable admissions are referred to physician advisors who also either approve the admission or question its necessity. The physician advisor then takes action to address the case.

Wide variation was observed in the actions taken by physician advisors. At one extreme, some advisors simply noted questionable cases and prepared for possible appeals with third parties. Others wrote or called the admitting physician and asked for additional documentation or discussion of the case. Still others worked with the admitting physician to justify the case or discharge the patient, and in certain instances they took the case to the relevant medical staff committee.

Irrespective of the defined role of the physician advisor, a consideration for programs is the relationship between the advisor and medical staff. Should the advisor be a regular member of the medical staff? on one hand, a physician advisor who is a member of the medical staff is in a difficult position, since judgments may affect future referrals, particularly for specialists. But on the other hand, physician advisors who are consultants might not be trusted and might be less effective in influencing behavior. At most hospitals, the advisor was a part-time appointment for a medical staff member, whose primary focus was on documentation of cases and the appeals process. In one hospital with a higher than desired level of payment denials, an outside consultant was employed to provided daily consultation to utilization reviewers and the medical staff. 


\section{Medical Staff and Administration Relations}

At the core of the utilization management process is some form of review of physicians' admitting, continuing stay, or discharge decisions, and any review will inevitably lead to tension and conflict between the medical staff and hospital administration. In a recent study of physician-hospital relations, hospital admissions policies and physician autonomy were two of the highest ranked areas in terms of adding stress to hospital-physician relations (16). What this means is that the design of hospital utilization management programs must acknowledge the potential trade off between revenue and maintaining medical staff goodwill.

In this context, it was not surprising to find a variety of policies on appropriate utilization management functions. A commonly found attitude among physicians was that they would do whatever is necessary to comply with utilization management, but not if it compromises their practice. At many hospitals the main focus of the review process was to meet regulatory requirements, to make sure that the i's are dotted and t's crossed in the chart. At these facilities, the expectation was that utilization management had little influence on medical practice.

At other hospitals, where utilization management was perceived to have had a more substantial influence, some persons suggested that they are not practicing as good medicine as they did before, due to the intimidation of physicians by the insurance carriers. Only somewhat sarcastically it was commented that "they are letting companies dictate how to practice medicine when they can't even make their own products well."

It was widely agreed that the most important component of effective utilization management was the quality and cooperation of the medical staff. However, not all hospitals can have the best and most enlightened physicians on their staff, and therefore utilization management and other administrative units must work more with their medical staff. Cooperation and diplomacy with the medical staff were universal components of effective utilization management. It was stated more than once that utilization management needs to "bend over backwards to avoid an adversarial environment."

\section{LESSONS LEARNED}

From our study of hospital utilization management programs, a set of knowledge was acquired which we believe bears consideration, even without substantial empirical investigation. While much of this knowledge does not lend itself to easy summarization, ten of the more important elements in predicting successful and cost-effective hospital utilization management programs are outlined below.

We believe that utilization management programs that are successful and effective are those which

1. Maintain a constant watch for new and potential regulations from government and private sector insurer groups and maintain an outward stance that is positive and proactive;

2. Perform comparative analyses of information available from external reviews of utilization outcomes and know generally how the results compare with those of competitiors;

3. Make continuous and detailed efforts to inform and update the medical staff on emerging regulations and requirements from insurers and the hospital's outcome experience;

4. Promote a high level of involvement with the medical staff leadership in developing hospital admission and discharge policies and policies related to chart completion;

5. Cultivate a high level of mutual trust and respect in the relationship between the administration and the medical staff and work well together in managing other areas of the hospital;

6. Have at least one person in a key leadership role on the medical staff interested in the hospital's utilization management experience and committed to helping the hospital and the medical staff work together in developing long-run, strategic responses;

7. Hire, train, and supervise utilization management staff who understand their role and the goals of the department in the larger context of the hospital's needs and its relationship with the medical staff;

8. Generate relevant information that flows well among managers and physicians responsible for quality assurance, infection control, risk management, medical records, patient accounts, discharge planning, and utilization management;

9. Encourage a friendly and positive relationship in working with physician office staffs on admission policies and precertification requirements; and

10. Have an aggressive program of appealing most, if not all, denied cases both to manage external relations and demonstrate support of internal staff and programs.

\section{References}

1. Mozes B, Halkin H, Katz A, et al. Reduction of redundant hospital stay through controlled intervention. Lancet 1987; 8539:968-979. 
2. Catchpole BL. A utilization management success story. Dimens Health Serv 1991;68:16-17.

3. Woodside JR, Bache R, Tonnesen AS, et al. Intensive focused utilization management in a teaching hospital: an exploratory study. Qual Assurance Util Rev 1991;6:47-50.

4. Carroll NW. Ford has another idea. Business and Health 1991;9: 79-82.

5. Feldstein PJ, Wickizer T, Wheeler JRC. The effects of utilization review programs on health care use and expenditures. $N$ Engl J Med 1988;318:1310-1314.

6. Latta VB, Keene RE. Use and cost of short-stay hospital inpatient services under Medicare, 1988. Health Care Financ Rev 1990;12:91-100.

7. Michigan Peer Review Organization. Memorandum. Plymouth, The Organization, 1990.

8. Longo DR, Ciccone KR, Lord JT. Integrated Quality Assess ment: A Model for Concurrent Review. Chicago, American Hospital Association, 1989:65-74.

9. Hurley RE, Michelman JE. Hospital response variation to the demands of preadmission certification. Qual Assurance Util Rev 1991;6:16-23.

10. Zusman J. Guidelines for the practice of utilization review: essential but lacking. Qual Rev Bull 1990;16:143-148.

11. Strumwasser I, Paranjpe NV, Ronis DL, et al. Reliability and validity of utilization review criteria: appropriateness evaluation protocol, standardized medreview instrument, and intensity-severity-discharge criteria. Med Care 1990;28:95-111.

12. Linton AL, Peachey DK. Utilization management: a medical responsibility. Can Med Assoc $J$ 1989;141:283-286.

13. Sutherland RW. Utilization management must be shared. Can Med Assoc $J$ 1989;141:287.

14. Connors EJ. Sisters of Merce Health Corporation's efforts to build the medical staff of the future. Frontiers of Health Services Management 1985;1:62-65.

15. Kennedy EK. Assertive Utilization Management For Nurses, Physicians and Hospital Administrators, p 59. San Mateo, MAGE Corporation, 1990.

16. Shortell SM. Effective Hospital-Physician Relationships. Ann Arbor, MI: Health Administration Press, 1991.

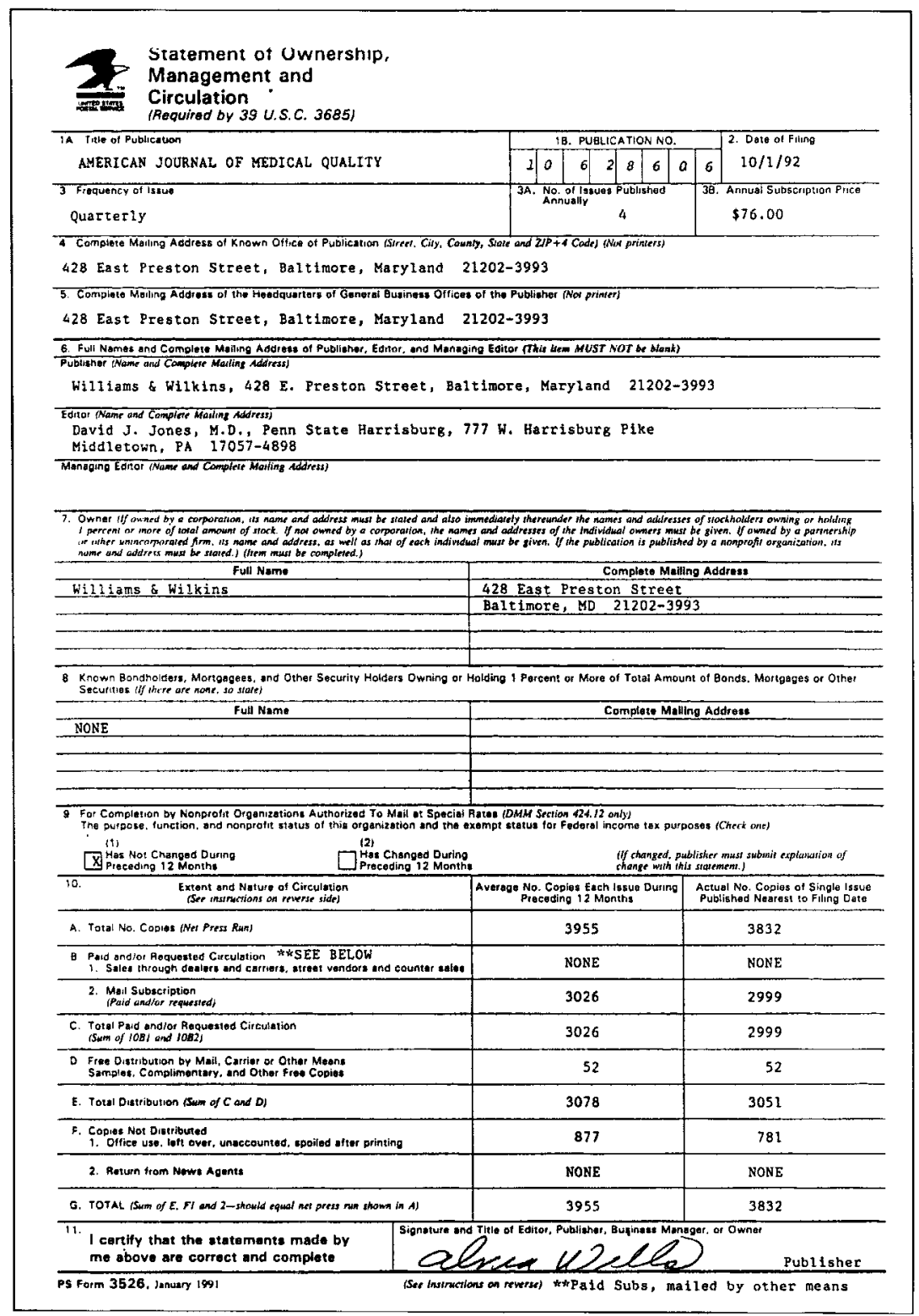

